\title{
Models for the development of multi-level gas supply systems
}

\author{
Nikolay Ilkevich ${ }^{1}$, Tatyana Dzyubina ${ }^{1,}$ *, Zhanna Kalinina ${ }^{1}$ \\ ${ }^{1}$ Melentiev Energy Systems Institute of Siberian branch of Russian Academy of Science (ESI SB RAS), department of pipeline and \\ hydraulic systems, Lermontov street 130, Irkutsk, Russia, 664033
}

\begin{abstract}
The article shows the relevance of hierarchical modeling in substantiating the optimal development of the gas industry in modern conditions. The questions of aggregation of calculation schemes of gas supply systems are considered. A set of mathematical models is proposed that allows us to consider it at three levels of the hierarchy, taking into account the improvement, refinement and detail of the information base being developed. Models are considered: 1) optimization of the structure of gas supply systems; 2) optimization of se-zone gas consumption, analysis and synthesis of reliability; 3 ) optimization of parameters of the main gaswire taking into account reliability.

The created information base on gas supply systems of the Russian Federation includes dynamics of development of demand for natural gas in the domestic and foreign markets, aggregated technical and technological, cost and reliability characteristics of system objects (main gas lines, fields, underground gas storage facilities) and forecasts of gas production.

On the basis of mathematical models, studies of the development of multi-level gas supply systems in Russia for the period up to 2030 are performed: the rates and directions of development of the gas transport structure, commissioning of new fields and optimal gas flows through the Russian Federation are substantiated. A systematic assessment of the means of regulating seasonal unevenness of gas consumption and the means of ensuring the reliability of the North-Western district of the Russian Federation was carried out.
\end{abstract}

\section{Introduction}

The unified gas supply system (UGSS) of Russia is a unique system of large size, which is not equaled in the world. The problem of hierarchical modeling of its optimal development began to be studied at the end of the last century $[1,2]$, and continues to be studied now, including in the ISEM SB RAS [2-4]. Questions of multilevel modeling are also raised abroad [5-17]. Various tasks of forecasting the world and national development of gas supply systems (GSS) are solved (gas flow models are usually used), each at its own hierarchical level. There are world energy [5-7] and gas models [8-14], models of the European $[15,16]$ and national market [17]. Gas flows, demand, production, gas prices, and the necessary new capacities of gas transport corridors and gas liquefaction plants are projected for different perspectives. Information exchange of data can be carried out between individual models of different hierarchical levels.

The size and complexity of the GSS, various aspects of their functioning and development make it necessary to consider them at different levels of the hierarchy, taking into account the improvement, refinement and detail of the information base being developed. Therefore, research in the field of multi-level modeling of development in the gas industry is an urgent task.
The object of research is the gas industry, which includes gas supply systems that supply consumers with hydrocarbon gases - the most important raw material resource for obtaining chemical products and environmentally friendly types of energy.

The article considers hierarchical modeling of optimal development of multi-level gas supply systems, including mathematical models of their development, models of reliability analysis and synthesis, and optimization of object parameters with regard to reliability.

\section{Aggregation of gas supply companies}

Aggregation of the calculation scheme is understood as modeling of the real gas supply scheme in an enlarged form [18]. Such a scheme should reflect the real scheme with a certain accuracy, preserving its required properties. The resulting aggregated scheme is characterized by a smaller number of nodes and connections, which makes it easier to analyze the results in order to develop the necessary solutions and use the information for calculations in mathematical models.

The GSS is represented as a directed graph and is considered as a set of three sub-systems: gas sources, main transport networks, and consumers.

The source objects are all enterprises that supply gas to the main transport network: integrated gas treatment plants, gas chemical complexes and underground gas storage facilities (UGS) that operate on gas extraction. A

\footnotetext{
$\overline{\text { * Corresponding author: tvleo@isem.irk.ru }}$
} 
gas producing enterprise is designated as an aggregated source unit, where gas production is determined by the total production of the fields.

Gas main transport enterprises consist of sections of main gas pipelines (MG), including the linear part (LP) and compressor stations (CS) located on it. Aggregated multiline MG are represented as single-line arcs, which are characterized by the total capacity of gas pipelines and the total length of all MG going from one node to another.

Consumer objects take gas from main gas pipelines, as well as UGS, if they are working for gas injection at the moment in question. Consumption nodes are aggregated on an administrative and geographical basis, with the Russian Federation's constituent entities serving as consumers. The demand for natural gas of the aggregated consumer is determined from the condition of equality of needs in the initial and aggregated schemes.

If the CS does not match the aggregated consumer node, it is designated as a branch node in the diagram. This node is necessary to correctly reflect the main gas flows in the diagram. The need for gas in the branch node is not set. The entire need of the subject is concentrated in the consumer node.

To determine the aggregated technical and economic characteristics of each arc and node of the aggregated calculation scheme, we use statistical data from PJSC Gazprom [19], and also take the original technical and economic information on existing gas production and gas transportation enterprises.
The final operation for forming the design scheme is "gluing" all aggregated schemes into one, "gluing" is carried out along the boundaries of the gas transportation enterprises. For example, a complex multi-line UGSS (Fig. 1) is presented as an aggregated calculation scheme. Existing large-scale projects of gas transport systems are superimposed on the aggregated existing scheme of the UGSS by the years of the planned periods. Thus, a redundant aggregated calculation scheme is created that reflects the stages of development of the GSS for the studied perspective (Fig. 2).

Based on the data [19], an information base is also being developed for multi-level modeling of the development of Russian gas supply systems for the period up to 2030 [4]. It shows the demand for gas in the nodes of the scheme, the upper limits on production and transport, as well as costs and coefficients showing the gas consumption for own needs and leaks. It also provides estimates of the dynamics of demand for natural gas in the Russian Federation and its export supplies (the state and prospects for the development of gas supply markets in the Russian Federation); technical and economic indicators for existing and new gas production enterprises and gas transportation systems.

The completed methodological developments allow us to set and solve complex tasks for the optimal development of gas supply systems in the future.

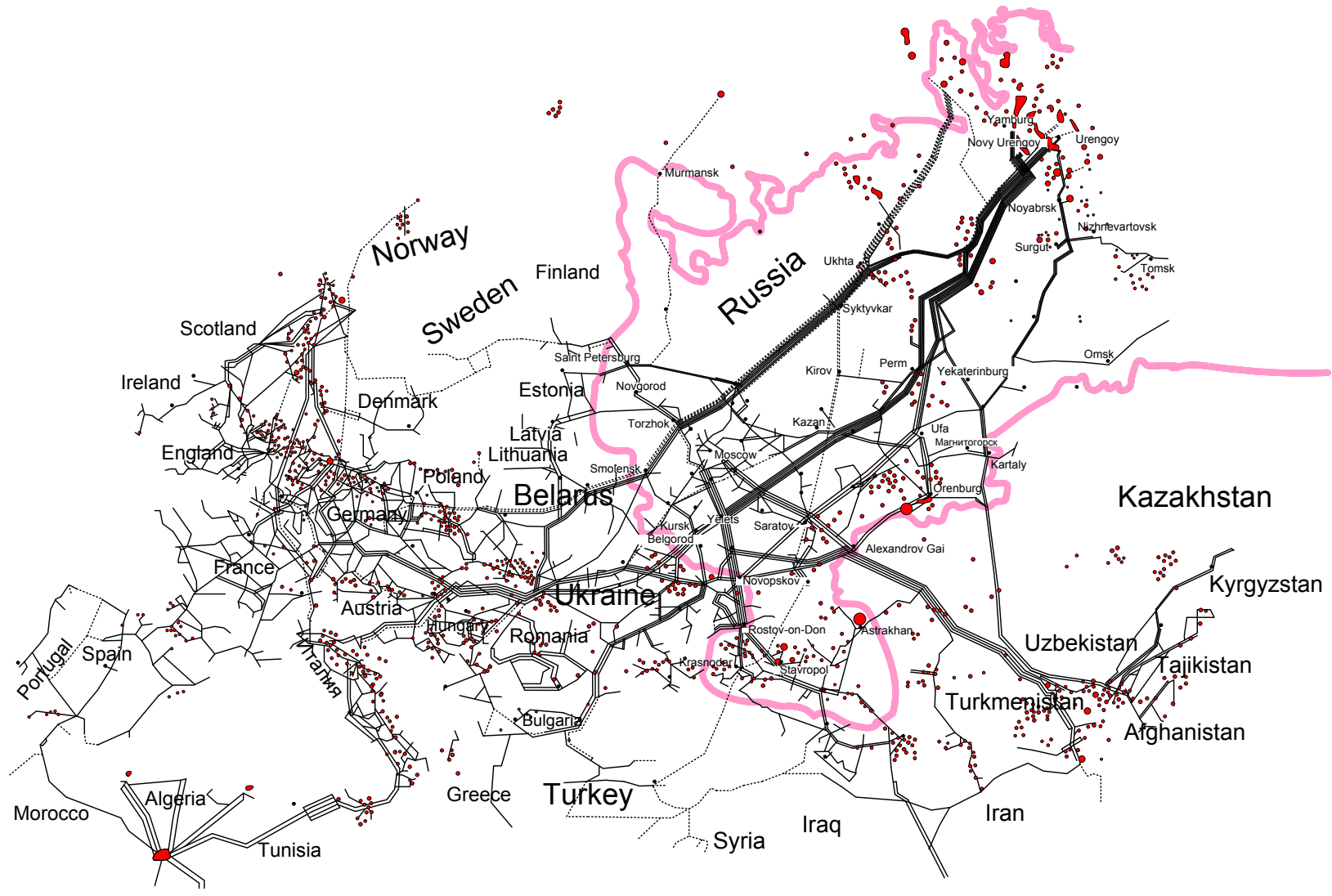

Fig. 1. Unified gas supply system. 


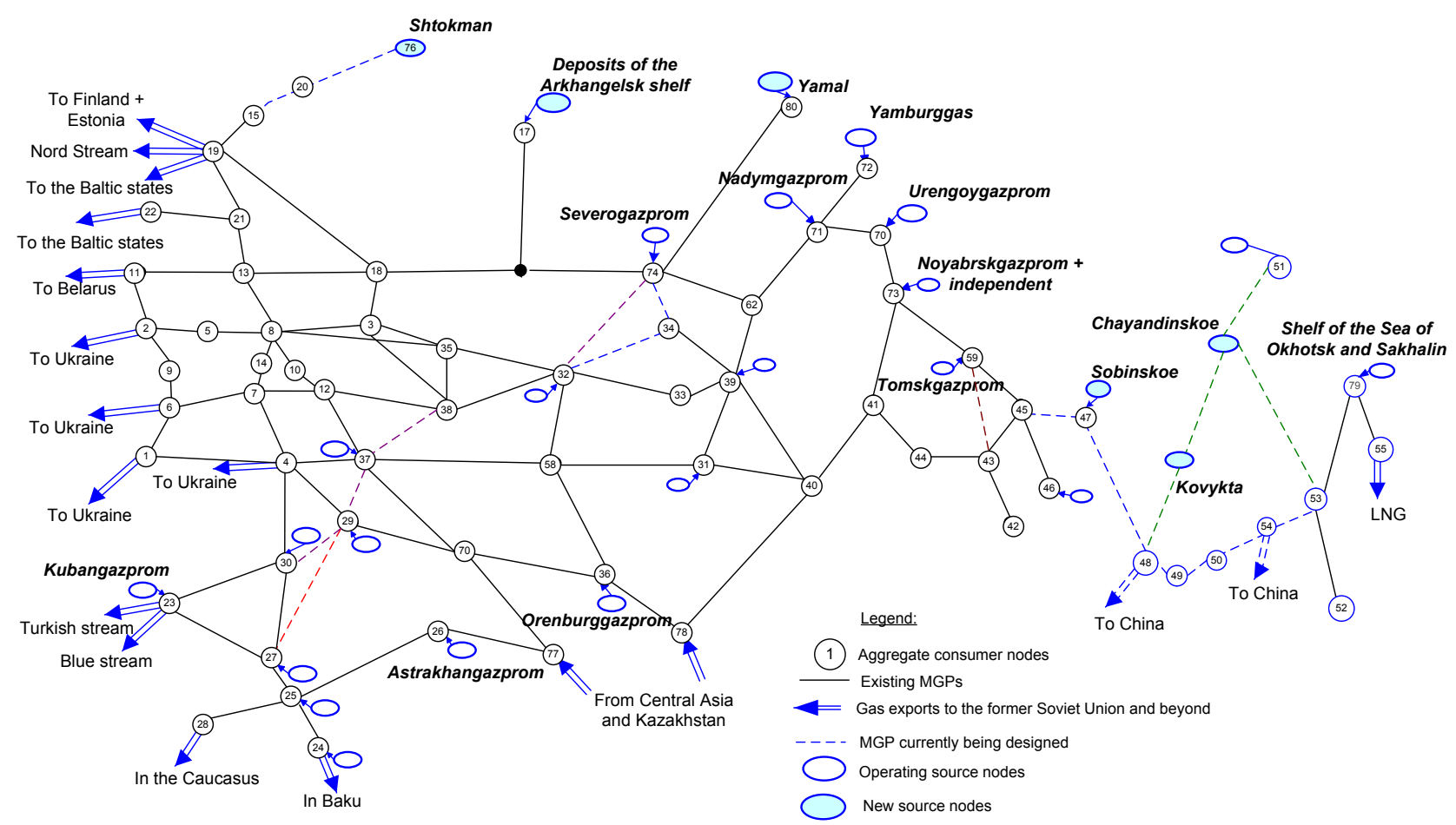

Fig. 2. Redundant aggregate calculation scheme of the GSS of the Russian Federation.

Models for the optimal development of the fuel and energy complex and other sectors of the economy

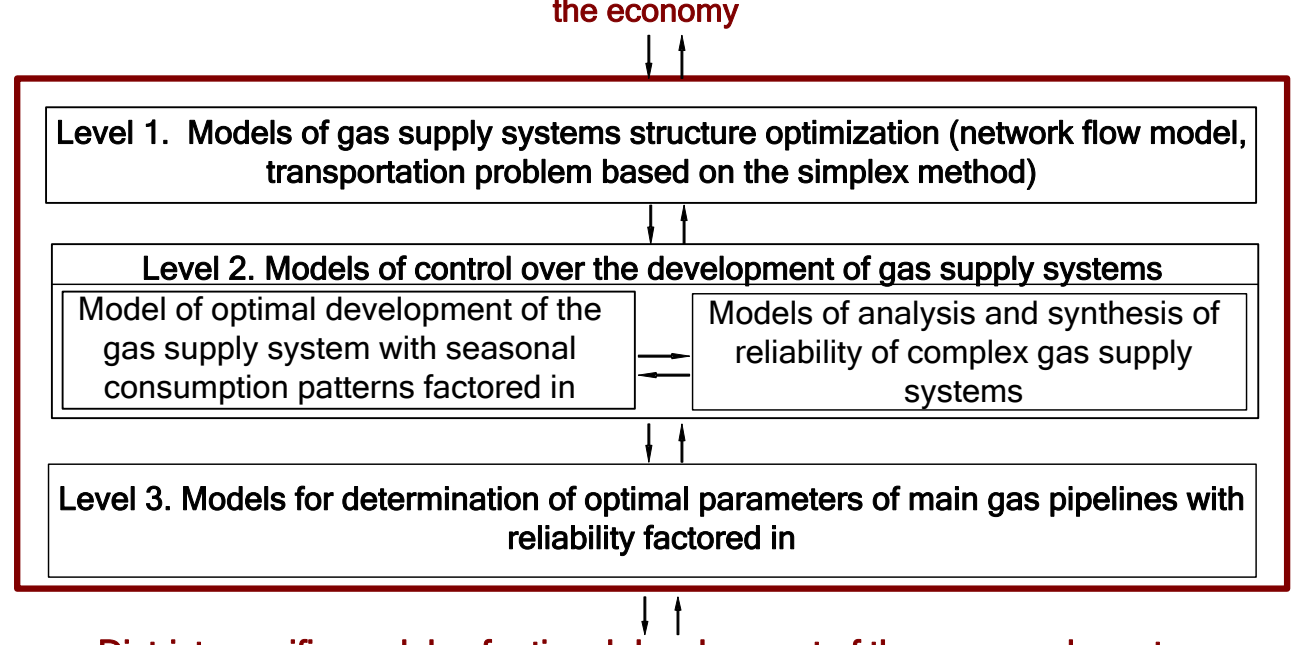

District-specific models of rational development of the gas supply system

Fig. 3. Models for solving problems of optimal development of gas supply systems.

\section{Aggregation Complex tasks of optimal development for the future}

Fig. 3 shows the models developed at ISEM SB RAS for solving problems of optimal development of gas supply systems and their interaction at three levels of consideration [4].

Model for optimizing the structure of the gas supply system. This network flow model allows you to find the optimal gas supply plan when there is a fixed demand for gas from consumers. It is solved at the first level of the hierarchy.
The generalized flow simulation problem is written as follows:

$$
\begin{gathered}
\sum_{(i, j)}\left(c_{i j} x_{i j}+k_{i j} y_{i j}\right) \rightarrow \min \\
\sum_{i} \lambda_{i j} x_{i j}-\sum_{i} x_{j i}= \begin{cases}-v, & j=s \\
0, & j \neq s, t \\
w, & j=t\end{cases} \\
l_{i j} \leq x_{i j} \leq d_{i j}+y_{i j}, \quad(i, j) \in U, \\
0 \leq y_{i j} \leq g_{i j}, \quad(i, j) \in U .
\end{gathered}
$$




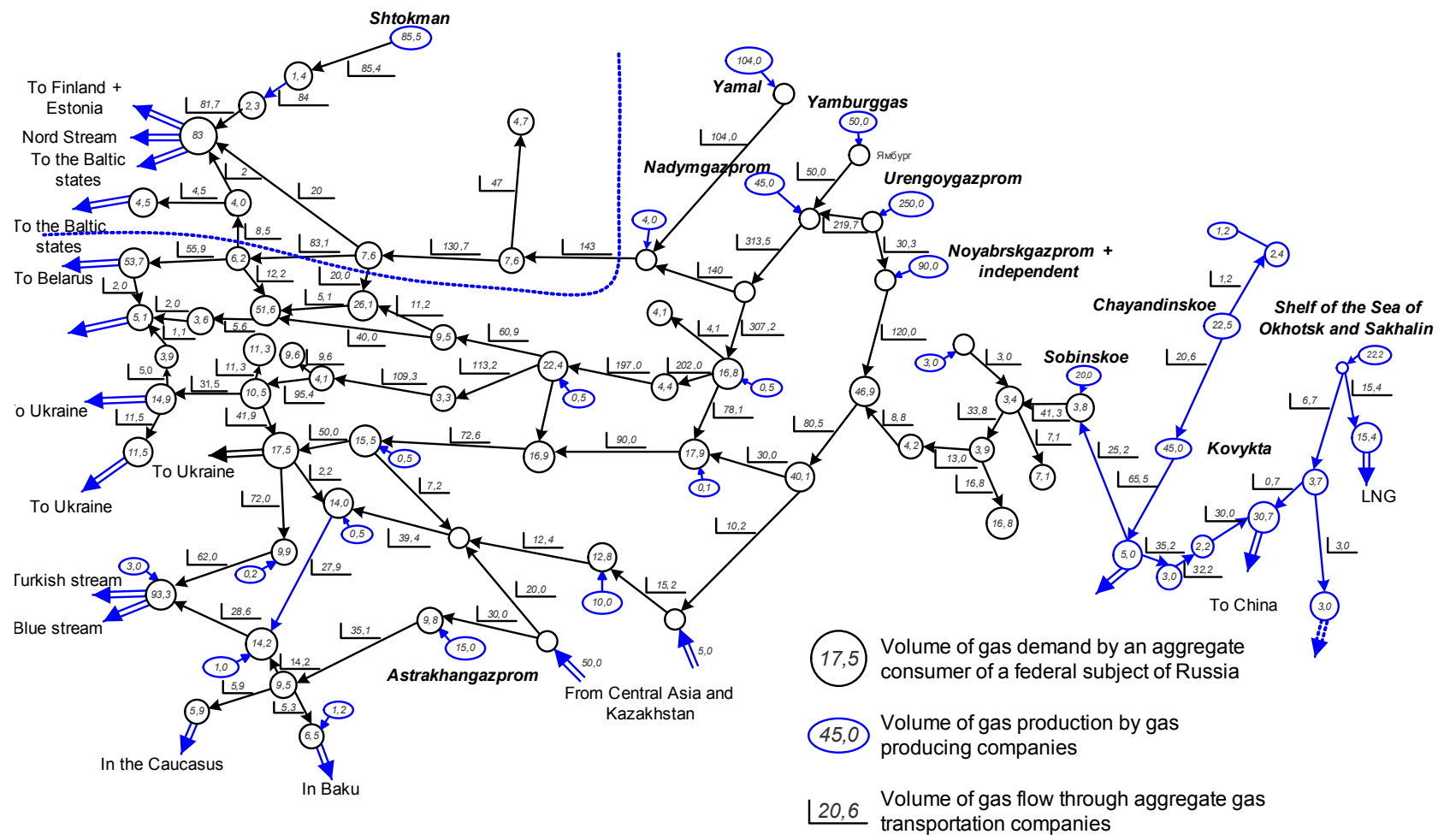

Fig. 4. Optimal volumes of gas production and transportation for the average scenario of consumption in the Russian Federation and export in 2030, billion cubic meters $\mathrm{m} /$ year.

As an optimality criterion, the minimum costs for the production, transport and delivery of gas to consumers are considered, the limitations are the production capacities of existing and new enterprises and the requirements to meet the minimum demand from consumers, provided that the balance of gas supply and withdrawal at the network nodes is maintained. This is the minimum cost flow problem, solved by the modified Basaker-Gowan algorithm [1].

Based on the data of the created information base, calculations were performed showing the optimal volumes of gas production and transportation for the average scenario of consumption in the Russian Federation and exports for 2020, 2025 and 2030. The calculation for 2030 is shown in Fig. 4, which shows the optimal volumes of gas production by gas producing enterprises and the volumes of gas flows through aggregated gas transmission enterprises.

The dotted line in Fig. 4, the scheme of gas supply to the Northwestern Federal District was highlighted. Using this diagram as an example, the detailed solutions of models of problems of lower levels of consideration will be shown.

A model for regulating the seasonal unevenness of gas consumption. Using this model, the solution obtained for the annual period at the top level of the hierarchy is detailed by seasons for summer and winter.
The model is a system of linear equations and inequalities that consistently describe the processes of production, transport, storage and consumption of gas by seasons:

$$
\begin{aligned}
& \sum_{i=1}^{n} \sum_{\tau=1}^{T}\left(c_{i \tau}^{P} x_{i \tau}^{P}+c_{i \tau}^{T} x_{i \tau}^{T}+c_{i \tau}^{x} x_{i \tau}^{x}+\sum_{l=1}^{L} c_{i \tau l}^{U} x_{i \tau l}^{U}+c_{i \tau}^{b} x_{i \tau}^{b}+u_{i \tau} z_{i \tau}\right) \rightarrow \min ; \\
& \sum_{\tau=1}^{T}\left(a_{i \tau}^{P} x_{i \tau}^{P}+a_{i \tau}^{T} x_{i \tau}^{T}+a_{i \tau}^{x} x_{i \tau}^{x}+\sum_{l=1}^{L} a_{i \tau l}^{U} x_{i \tau l}^{U}+z_{i \tau}\right)=\sum_{\tau=1}^{T}\left(x_{i \tau}^{-T}+a_{i \tau}^{b} x_{i \tau}^{b}+b_{i \tau}\right), \\
& 0 \leq a_{i \tau}^{P} x_{i \tau}^{P} \leq d_{i \tau}^{P} ; 0 \leq a_{i \tau}^{T} x_{i \tau}^{T} \leq d_{i \tau}^{T} ; 0 \leq a_{i \tau}^{x} x_{i \tau}^{x} \leq d_{i \tau}^{x} ; 0 \leq a_{i \tau}^{U} x_{i \tau}^{U} \leq d_{i \tau}^{U}
\end{aligned}
$$

The model can take into account constraints on limited resources: fuel oil $\left(d^{f}\right)$, coal $\left(d^{c}\right)$, total capital investment $(k)$ and metal $(M)$.

$$
\begin{gathered}
0 \leq \sum_{i=1}^{n} \sum_{\tau=1}^{T} \sum_{l=1}^{L} a_{i \tau l}^{U} x_{i \tau l}^{U} \leq d^{f} ; \\
0 \leq \sum_{i=1}^{n} \sum_{\tau=1}^{T}\left(\sum_{l=1}^{L} a_{i \tau l}^{U} x_{i \tau l}^{U}+a_{i \tau l}^{b} x_{i \tau l}^{b}\right) \leq d^{c} ; \\
0 \leq \sum_{i=1}^{n} \sum_{\tau=1}^{T}\left(k_{i \tau}^{P} x_{i \tau}^{P}+k_{i \tau}^{T} x_{i \tau}^{T}+k_{i \tau}^{x} x_{i \tau}^{x}+\sum_{l=1}^{L} k_{i \tau l}^{U} x_{i \tau l}^{U}+k_{i \tau l}^{b} x_{i \tau l}^{b}\right) \leq k ; \\
0 \leq \sum_{i=1}^{n} \sum_{\tau=1}^{T} \mu_{i \tau} x_{i \tau}^{T} \leq M,
\end{gathered}
$$



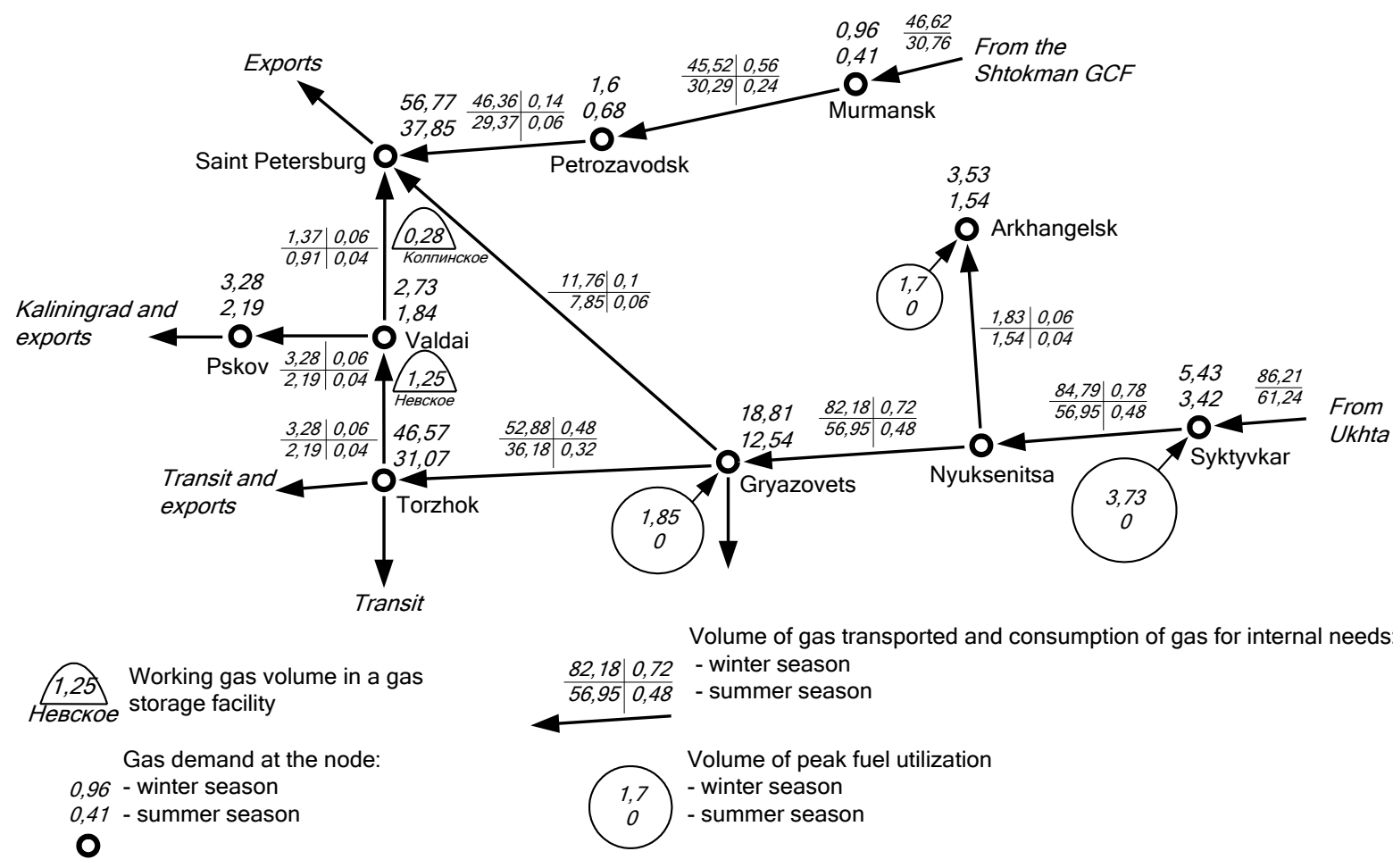

Volume of gas transported and consumption of gas for internal needs

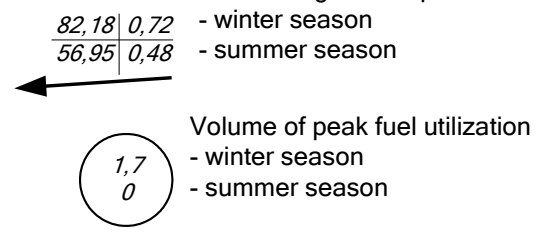

Fig. 5. Regulation of seasonal irregularity of gas supply in the Northwestern Federal District in 2030, million tons of fuel equivalent.

The criterion is the minimized function of costs for production, transportation, storage and use of gas; the following expression shows the condition for equality of flows of production, transport, storage and consumption of gas; then there are restrictions on gas flows; for investment; on metallurgy.

As a result of solving this problem by standard methods of linear programming by seasons of the year, the productivity of fields, gas transmission enterprises and underground gas storage facilities is determined.

On the model of regulation of seasonal unevenness, a detailed scheme of gas supply to the North-Western Federal District in 2030 was calculated (Fig. 5). It shows rational volumes of transported gas and gas consumption for own needs in winter and summer periods, volumes of storage and use of gas in underground storage facilities and volumes of peak fuel use.

Reliability synthesis model for a complex gas supply system. This detailed solution is the initial information for modeling the rational reliability of the GSS. For this, a two-stage methodological approach is proposed, in which the following tasks are solved [20]:

Stage 1. Determination of equivalent reliability characteristics for main gas pipelines, fields and underground gas storages, as well as for structures storing gas and other fuel reserves at consumers, allowing them to replace gas. For this, the models for analyzing the reliability of GSS facilities are used.

Stage 2. Optimization of gas supply system backup means. The problem of determining the optimal combination of redundancy methods that satisfy in each node of the design scheme the balances of incoming and outgoing mathematical expectations of the productivity of objects that provide consumers with volumes of gas and reserves of other fuel with a given reliability and given restrictions is formulated as follows:

$$
\begin{aligned}
& \sum_{(i, j) \in U}\left(c_{i j} x_{i j}+k_{i j} y_{i j}\right)+p_{j} z_{j} \rightarrow \min \\
& \sum_{i \in \Gamma_{j}^{+}}\left(\lambda_{i j} x_{i j}+\pi_{i j} y_{i j}\right)+\alpha_{j} z_{j}-\sum_{j \in \Gamma_{j}^{-}} x_{j i}=\left\{\begin{array}{c}
-Q, j=s ; \\
0, j \neq s, t ; \\
B, j=t .
\end{array}\right. \\
& 0 \leq x_{i j} \leq d_{i j} ; \quad 0 \leq y_{i j} \leq d_{i j}^{r}-d_{i j} ; 0 \leq z_{j} \leq Z_{j} .
\end{aligned}
$$

The minimum of the objective cost function is considered as a criterion. Shows the balances of the arrival and departure of the capacities of objects with existing redundancy and with additional reserve funds for these objects, as well as taking into account reserves of reserve fuel. For each node, a balance of incoming and outgoing capacities must be observed (Kirchhoff's first law). The last line shows the two-way performance limits for objects. The problem is solved by standard linear programming methods. 


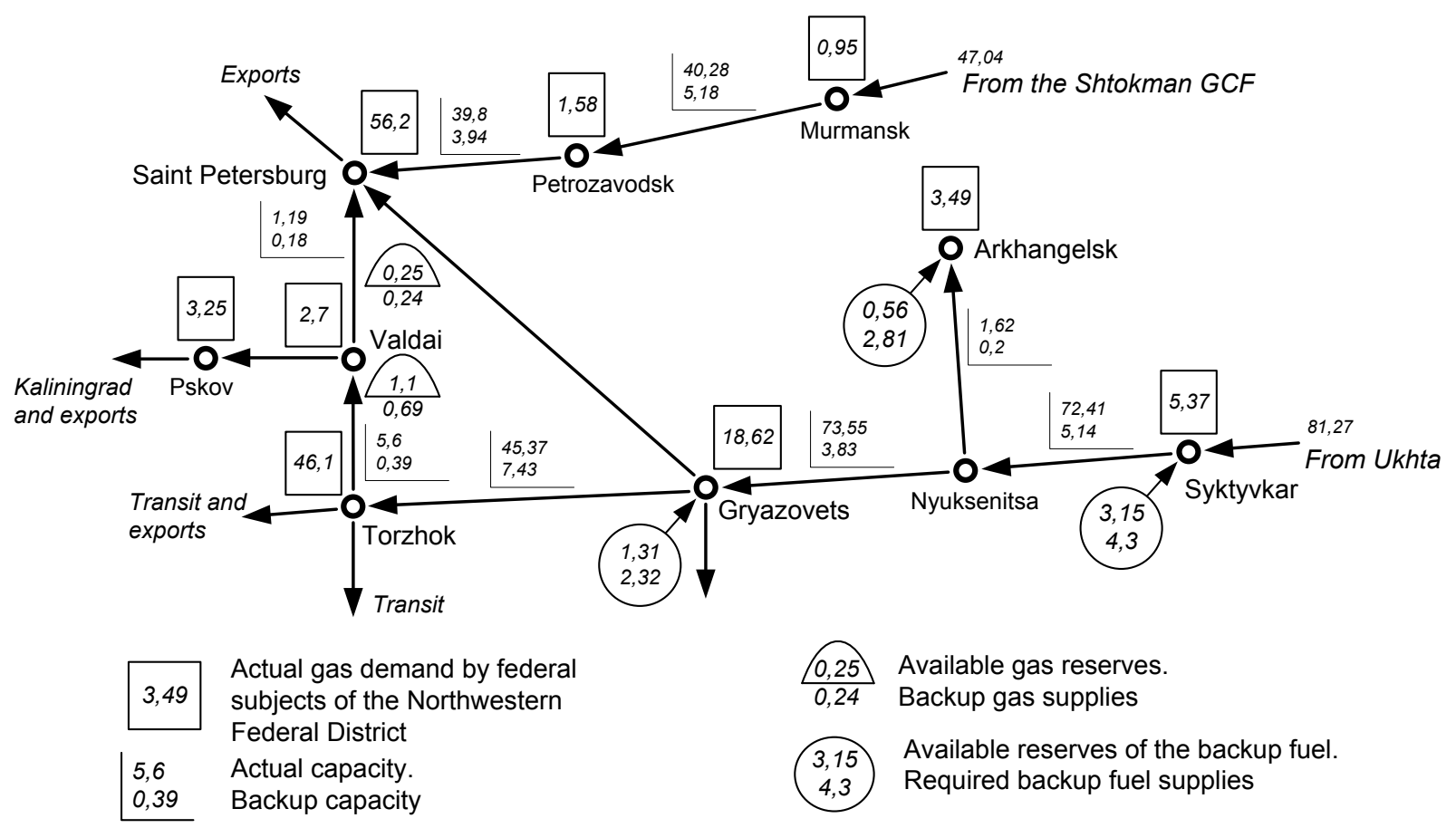

Fig. 6. Optimal supply redundancy of the gas supply system of the Northwestern Federal District in the winter period of 2030.

In fig. 6 shows the results of optimization of the system reliability of the Northwestern Federal District in the winter period for 2030, which detail the solution to the problem of seasonal unevenness. To ensure the actual gas demand in the subjects of the Northwestern Federal District with a supply ratio of 0.99 , it is necessary to create additional reserve productivity in addition to the actual productivity of the elements, as well as reserves of reserve fuel for a number of consumers in the district, as shown in Fig. 6.

Model for determining the optimal parameters of the main gas pipeline, taking into account reliability. The General process of selecting optimal MG parameters involves:

1. Alternative consideration of ways of development for the future of the object under consideration.

2. Analysis of its reliability.

3. Optimal choice of a rational option based on the calculation of technical and economic characteristics and integrated reliability indicators.

The problem of determining the rational parameters of the designed MG, taking into account reliability, is generally formulated as follows.

Based on the average daily MG capacity $(Q)$, its technical and process $(T)$, reliability $(N)$, and technical and economic performance indicators $(E)$, the basic scheme of the MG and redundant final backup methods ( $r$ ) to determine the diameters of a line for line pipes, the number of CSs and installed GPUs (gas pumping unit) that would maximize income $Z$ from gas sales, provided that the specified reliability standard of $P^{*}$ of gas supply is to be complied with.

$$
\begin{aligned}
& Z=f(T, N, E, r) \rightarrow \max \\
& P=y(Q, N, r) \geq P^{*}
\end{aligned}
$$

The average daily calculated capacity $(Q)$ is determined based on the annual calculated capacity of the
MGP taking into account the coefficient of nonuniformity of gas consumption. For MGs without underground gas storage (UGS) facilities at the consumers' end, it is typically assumed to be 0.85 , while for branch lines of the trunkline it is 0.75 .

Technical and process indicators $(T)$ are as follows: the MG length, the list of the number of lines and corresponding diameters, the list of standard sizes of rated GPU capacity (the number of considered options for LPs and CSs).

Reliability indicators $(N)$ are understood as the rate of failure and recovery of LPs and GPUs. As a normative reliability indicator of gas pipeline $P^{*}$, we take reliability factor $K_{n}$. Its current value $(P)$ is the ratio of the mathematical expectation of performance to its rated value:

$$
K_{n}=\frac{M[Q]}{Q_{n}} .
$$

Technical and economic indicators (e) are defined as: specific annual operating costs and capital investment in LP MG; specific annual operating costs and specific annual capital investment, proportional to the installed capacity of the compressor station; specific metal investment.

As a result of solving this problem of synthesis (optimization) of the structural reliability of the designed MG, the following parameters are determined: the number of pipeline threads; the corresponding optimal diameters; the number of CS; the number and length of linear sections; the number of working and reserve GPU on each CS; the optimal nominal capacity of the GPU; metal deposits in the LP.

The number of all possible variants of the designed $M G$ is equal to the product of the numbers of options LP MG and sizes of GPU for KS and the maximum number of backup 
units to cs, which should not exceed number of operating units.

The formulated problem can be considered as a combinatorial optimization problem. Engineering research experience shows that the number of options for the development of the gas pipeline is relatively small, and all of them can be viewed by ordinary search.

Table shows the results of parameter optimization taking into account the reliability of the Kovyktinskoye gas transmission system - Irkutsk - Beijing.

Table. Optimization of gas transportation system parameters Kovykta GCF - Irkutsk - Beijing, with reliability factored in.

\begin{tabular}{|c|c|c|}
\hline Parameter & Kovykta GCF - Irkutsk & Irkutsk-Beijing \\
\hline Top & 25 & 25 \\
\hline Bottom & 20 & 20 \\
\hline Diameter and number of lines & $1220 \times 2+1420$ & 1420 \\
\hline Pipeline length, km & 470 & 2170 \\
\hline Number of CSs & $2(3)^{*}$ & 16 \\
\hline Number of installed GPUs & 9 & 6 \\
\hline Number of backup GPUs & 3 & 3 \\
\hline GPU type & GPA-Ts-16 & GPA-Ts-16 \\
\hline Resulting reliability & 0.978 & 0.974 \\
\hline Capacity of a single CS & 128.5 & 82.9 \\
\hline $\begin{array}{l}\text { Specific capital expenditures } \\
\text { per } 1 \mathrm{~km} \text {, million doll. }\end{array}$ & 2.35 & 2.32 \\
\hline Net present value, mln. USD & 36,035 & 25,263 \\
\hline Internal rate of return, $\%$ & 58.9 & 25.2 \\
\hline Year of loan repayment & 7 & 7 \\
\hline Metal inputs, thous. tons & 886 & 1634 \\
\hline
\end{tabular}

\section{Conclusions}

1. Taking into account the General issues of aggregation of enterprises of gas supply systems, hierarchical modeling of optimal development is considered, namely: 1) optimization of the GSS structure; 2) optimization of seasonal gas consumption, analysis and synthesis of reliability; 3) optimization of object parameters taking into account reliability.
2. Based on the proposed method of multi-level modeling of the gas supply system development, optimization calculations were made: gas production and transport volumes for the average scenario of consumption in the Russian Federation, rational seasonal unevenness of gas consumption in the North-Western Federal district, gas supply system reservation in the North-Western Federal district in winter, optimal parameters of the Kovyktinskoye GCM - Beijing mg. 


\section{References}

1. M. G. Sukharev, E. R. Stavrovsky, E. E. Bryanskikh, Optimal development of gas supply systems (Nedra, Moscow, 1981)

2. N.I Ilkevich,., Efremov V. A., A. P. Merenkov, Coordination of General energy solutions with the development of a Unified gas supply system, Math. Model. Optimiz. (Nauka, Novosibirsk, 1992)

3. N.I Ilkevich,., A. P. Merenkov, Multilevel modeling and coordination of tasks for the development of gas supply systems, Meth. Control Phys. Techn. Syst. (Nauka, Novosibirsk, 1995)

4. N.I Ilkevich, T.V. Dzyubina, Zh. V. Kalinina. Multilevel modeling of gas supply systems development (Nauka, Novosibirsk, 2014)

5. World energy model documentation 2016 version. Address of the Internet resource: http://www.iea.org/media/weowebsite/2016/WEM_Docu mentation_WEO2016.pdf

6. World Energy Projection System Plus: Global Activity Module (GLAM) Internet resource address: https://www.eia.gov/outlooks/ieo/weps/ documenttation/pdf/wepsplus2016_globalactivitymodule.pdf

7. The European side of the ledger. Address of the Internet resource:

http://energypolicy.columbia.edu/sites/default/files/The $\% 20$ European\%20Side\%20of\%20the\%20Ledger_Ameri can $\% 20$ Gas $\% 20$ to $\% 20$ the $\% 20$ Rescue $\% 3$ F.pdf

8. Subscribe to Nexant Thinking: World Gas Model Internet

Address pecypca:www2.nexant.com/brochure/world-gas-model 9. World Gas Model Address of the Internet resource: http://server.nexant.com/ecc/docs/WorldGasModel.pdf 10. Licensing the World Gas Model Address of the Internet resource: http://server.nexant.com/ecc/docs/World Gas Model Licensing Flyer_May2011.pdf

11. R. Egging, F. Holz, S.A. Gabriel, The World Gas Model: A Multi-Period Mixed Complementarity Model for the Global Natural Gas Market, Energ. Elsevier, , 35 10 (2010)

12. D. M. Nesbitt, Scientific Solutions for Complex Decision Problems Challenging Senior Management (2005). Address of the Internet resource: http://web.stanford.edu/class/

msande290/WarnersCourse

312005.pdfDeloitteMarketPoint. World Crude Oil and Refined Product Modeling [electronic resource] / / Deloitte Center for Energy

13. Solutions. Deloitte Development. (2011). - address of the Internet Resource: http://www.deloitte.com/assets/Dcom-UnitedStates/Local Assets/ Documentts/Energy_us_er/us_er_MarketPointWorldOil

ModelTechnicalbro-chure_081011.pdf

14. Global $L \overline{N G}$ Outlook (2012). http://www.marinemoney.com/sites/all/themes/marinem oney/forums/MMWeek12/presentations/wednesday/LN G 2.00 Choi. pdf

15. Y. Smeers. Gas models and three difficult objectives, (2009)

http://webdoc.sub.gwdg.de/ebook/serien/e/CORE/dp200 8_9.pdf

16. M. Martinez, M. Paletar, H. Hecking. The 2014 Ukrainian crisis:

http://www.ewi.unikoeln.de/fileadmin/user_upload/Instit ut/Startseite_Text/The_2014_Ukrainian_crisis_Europes _increased_security_position.pdf

17. U.S. Energy Information Administration https:/www.eia.gov/out-looks/aeo/overview/

18. Zh. V. Kalinina, N. I. Ilkevich, Methodological approach to aggregation of the unified gas supply system scheme, Pipel. Energy. Sys. (Nauka, Novosibirsk, 2014) 19. Gas industry. Economics and statistics review of $O A O$ "Gazprom" (2012)

20. N.I Ilkevich, T.V. Dzyubina, Zh. V. Kalinina, N. V Surnin, Optimization of development of gas supply systems in Russia for the period up to 2030. News of A. S. "Energy" 1 (2018).. 